\title{
Power and commutator structure of groups
}

\section{David Shield}

Let $G$ be a group with a normal subgroup $H$ which has index in $G$ equal to a power of a prime $p$, and which is nilpotent and has exponent a power of $p$. Baumslag [1] has shown that such a group $G$ is nilpotent; and that, conversely, if a non-trivial wreath product is nilpotent, then it satisfies the description above with $H$ as its base group. The main result of this thesis is an upper bound on the nilpotency class of $G$ in terms of parameters of $H$ and $G / H$. This bound is attained whenever $G$ is a wreath product and $H$ is its base group; thus the question of the nilpotency class of a wreath product (see Liebeck [3], Scruton [4]) is answered.

The answer involves a descending central series of subgroups, here called the cpp-series, previously defined independently by Jennings [2] and Zassenhaus [5]. Corresponding to an arbitrary group $G$ and a prime $p$, this is the lowest series

$$
G=\pi_{1}(G) \supseteq \pi_{2}(G) \supseteq \cdots \supseteq \pi_{i}(G) \supseteq \cdots
$$

such that for all positive integers $i$ and $j$,

$$
\left[\pi_{i}(G), \pi_{j}(G)\right] \subseteq \pi_{i+j}(G) \text { and }\left(\pi_{i}(G)\right)^{p} \subseteq \pi_{i p}(G)
$$

If for some positive integer $v, \pi_{v}(G) \supset \pi_{v+1}(G)=\{1\}$, then $G$ is cpp-nilpotent with cpp-class $v$. The cpp-series seems in several ways to be more "natural" in the present context than does the lower central series.

Received 16 June 1976. Thesis submitted to the Australian National University, January 1976. Degree approved, July 1976. Supervisor: Dr M. F. Newman. 
These are two of several central series related in varying ways to the $p$-power structure of a group, whose definitions arise from the investigations in Chapter $I$ into relationships in a group between the operations of commutation, raising to the $p^{\text {th }}$ power, and multiplication.

Chapter 2 contains a construction of a basis for a finite p-group, such that the standard form of an element exhibits its position in the cpp-series.

In Chapter 3, upper bounds on the nilpotency class and cpp-class of group extensions are calculated, and shown to be equal to lower bounds on the corresponding classes of wreath products. Let $J$ be a finite $p$-group of cpp-class $d$; for $1 \leq v \leq d$ suppose that $\left|\pi_{v}(J)\right|=p^{m(v)}$, and set

$$
a=1+(p-1) \sum\{m(v): 1 \leq v \leq d\}
$$

and $b=(p-1) d$.

From the results of Jennings [2], it can be seen that $C_{p}$ wr $J$ has nilpotency class $a$. More generally for arbitrary positive integer $s$, $C_{p}$ wr $J$ has nilpotency class $a+(s-1) b$. This is in turn a special case of the full result: if $H$ is a nilpotent p-group of class $c$, such that for $1 \leq w \leq c, p^{s(w)}$ is the maximum order of a commutator of weight $w$ in $H$, then $H$ wr $J$ is nilpotent of class precisely

$$
\max \{a w+b(s(w)-1): 1 \leq w \leq c\} .
$$

If the cpp-class of $H$ is $v$, then the cpp-class of $H$ wr $J$ is $a v$. Note that the values of $v$, of $c$, and of $s(w)$ for $1 \leq w \leq c$ are the same for $H$ as for an arbitrary direct power of $H$, such as the base group of $H$ wr $J$.

The main results of Chapter 3 are applied in Chapter 4 to show that if certain restricted Burnside groups (whose existence is an open question) do in fact exist, then they are very large, and have such high nilpotency class as to make it improbable that they can be computed by presently foreseen techniques. 


\section{References}

[1] Gilbert Baumslag, "Wreath products and p-groups", Proc. Cambridge Philos. Soc. 55 (1959), 224-231.

[2] S.A. Jennings, "The structure of the group ring of a p-group over a modular field", Trans. Amer. Math. Soc. 50 (1941), 175-185.

[3] Hans Liebeck, "Concerning nilpotent wreath products", Proc. Cambridge Philos. Soc. 58 (1962), 443-451.

[4] Teresa Scruton, "Bounds for the class of nilpotent wreath products", Proc. Cambridge Phizos. Soc. 62 (1966), 165-169.

[5] Hans Zassenhaus, "Ein Verfahren, jeder endlichen $p$-Gruppe einen LieRing mit der Charakteristik $p$ zuzuordnen", Abh. Math. Sem. Univ. Hamburg 13 (1940), 200-207. 